\title{
Anonymous Multi-Receiver Public Key Encryption Based on Lucas Sequences
}

\section{L houssain El Fadil $^{1}$, Abdelkader MOUMEN ${ }^{2}$ and Mohamed BOUYE ${ }^{3}$}

\author{
${ }^{1}$ Department of Mathematics, King Khalid University. \\ Faculty of Sciences, B.P. 1000, Abha, Kingdom of Saudi Arabia. \\ ${ }^{1}$ Polydisciplinary Faculty of Ouarzazat \\ P.O. Box 638, Ouarzazat-Morocco. \\ lhouelfadil2@gmail.com \\ ${ }^{2}$ Department of Mathematics, King Khalid University. \\ Faculty of Sciences, B.P. 1000, Abha, Kingdom of Saudi Arabia. \\ abdelkader.moumen@gmail.com \\ ${ }^{3}$ Department of Mathematics, King Khalid University. \\ Faculty of Sciences, B.P. 1000, Abha, Kingdom of Saudi Arabia. \\ medeni.doc@gmail.com
}

\begin{abstract}
Multi-receiver encryption enables a sender to encrypt a message and transmit the ciphertext to a set of authorized users, while no one out this group of authorized users can decrypt the message. Multi-receiver encryption is of great importance in many sectors such as broadcast communication, cloud computing, wireless communications, networking applications, e-voting, lottery, and medical applications. This paper proposes an efficient multi-receiver public key encryption scheme based on Lucas sequences. The results of the computational analysis show that, projected scheme is better against renown attacks and prevailing anonymous multireceiver algorithms.
\end{abstract}

Keywords: Lucas sequences, public key cryptosystems, Multi-receiver encryption scheme, Chinese Remainder Theorem, Anonymity.

\section{Introduction}

The goal of the multi-receiver encryption is to securely transmit any message to all authorized receivers via insecure channels. There are several proposed multi-receiver encryption schemes in the literature based on different technical [18, 7, 1, 14, 19, 21, 4, 9, 11].

In their paper Smith and Lennon [17], were the first to introduce linear sequences in cryptography in 1993. They suggested that the second order linear sequences could be used instead of the standard exponentiation used in RSA. Two years later, Chi-Sung et al has shown that the security 
of Lucas functions is polynomial-time equivalent to the generalized discrete logarithm problems [2]. In 2012, El Fadil proposed a cryptosystem based on second order linear sequences in which semantic security is ensured [10]. Moreover, as linear sequences are not multiplicative, the main advantage of Lucas cryptosystems is that they are not formulated in terms of exponentiation. This would make them invulnerable to various well known attacks that threaten the security of more traditional cryptosystems like RSA and Diffie Hellman [5]. However, many current networking applications involve multiple receivers and one-to-one type of encryption is not appropriate for such type of applications. The main motivation of multiple receivers is to secure that group communications which getting popularity in different domains.

In this paper, we propose a novel multi-receiver public key encryption scheme based on Lucas sequences. From analysis results, it is clear that our approach is more efficient and satisfying anonymity of receivers as compared to existing schemes. Section 2, consists of brief description of the second order linear sequences ("Lucas sequences") and their cryptography properties. After that, we present a new approach in Section 3 with security and computation analysis. Finally, we give our conclusion in Section 4.

\section{Second order linear sequences ("Lucas sequences")}

In this section, the main cryptographic properties of Lucas sequences are studied. A computational method to evaluate the $k^{t h}$ term are given, together with an analysis of its computational cost.

Throughout this section :

- $p$ is a prime integer.

- $\mathbb{F}_{p}=\mathbb{Z} / p \mathbb{Z}$ the finite field of $p$ elements.

- $(a, b) \in \mathbb{Z}^{2}$ such that $b \equiv 1(\bmod p)$.

- $f(X)=X^{2}-a X+b$ is primitive polynomial over $\mathbb{F}_{p}[X]$.

- $A=\mathbb{F}_{p}[X] /(f(X))$.

- $\alpha=\bar{X}$ the class of $X$ modulo the principal ideal of $\mathbb{F}_{p}[X]$ generated by $f(X)$.

- For every $x \in A, l_{x}$ be the linear map of $A$ defined by $l_{x}(y)=x y$.

- $T(x)=\operatorname{Tr}\left(l_{x}\right)$ the trace of $x, \operatorname{Tr}\left(l_{x}\right)$ the trace of the linear map $l_{x}$.

- $N(x)=\operatorname{det}\left(l_{x}\right)$ the norm of $x, \operatorname{det}\left(l_{x}\right)$ is the determinant of the linear map $l_{x}$.

- Define the sequence $s(a)$ as follows :

$$
k \in \mathbb{Z}, s_{k}(a)=T\left(\alpha^{k}\right) \text { for every integer. }
$$

Remark 2.1 Since $f(\alpha)=0$ and the map trace is linear, it follows that:

$$
s_{k+2}(a) \equiv a s_{k+1}(a)-s_{k}(a)(\bmod p) \text { modulo } p .
$$

So, $s(a)$ is a second order linear sequence (Lucas sequence), called the characteristic sequence generated by $a$.

Remark 2.2 Let $l_{k}$ be the endomorphism of $A$ defined by :

$$
\begin{array}{ccc}
l_{k}(x): A & \longrightarrow & A \\
x & \longmapsto & l_{k}(x)=\alpha^{k} x
\end{array}
$$

$M_{k}$ its matrix with respect to the basis $(1, \alpha)$.

$$
\begin{aligned}
& \text { Then } M_{0}=\left(\begin{array}{ll}
1 & 0 \\
0 & 1
\end{array}\right) \text { and } M_{1}=\left(\begin{array}{cc}
0 & -1 \\
1 & \bar{a}
\end{array}\right) \text {. } \\
& \text { Thus, } s_{0}(a) \equiv 2(\bmod p) \text { and } s_{1}(a) \equiv a(\bmod p) \text {. }
\end{aligned}
$$




\subsection{Cryptographic properties}

The cryptographic applications of Lucas sequences are listed in [16, 15]. To make this concept easy for the reader, we present some of these results in a more accessible form and without proofs.

1. For every integer $k, s_{k}(a) \equiv \alpha^{k}+\alpha^{-k}(\bmod p)$ and $s_{k}(a) \equiv s_{-k}(a)(\bmod p)$.

2. For every integers $k$ and $e, s_{e}\left(s_{k}(a)\right) \equiv s_{k e}(a)(\bmod p)$.

3. Let $a \in \mathbb{Z}$ such that $p$ does not divide $a^{2}-4$.

If $f(X)$ is a primitive polynomial over $p$, then $\pi=p+1$ is the period of $s(a)$. More general if $\left(\frac{a^{2}-4}{p}\right)=-1$, then $f(X)$ is irreducible. So $N(\alpha)=\alpha^{1+p}=1$. Thus the period $\pi$ of $s(a)$ divides $p+1$. If $\left(\frac{a^{2}-4}{p}\right)=1$, then $f(X)$ is reducible. The fact that $A \simeq \mathbb{F}_{p} \times \mathbb{F}_{p}$ implies that the period $\pi$ of $s(a)$ is $p-1$, where $(\overline{\bar{p}})$ is the Legendre symbol.

For the two first points, if $f(X)$ is irreducible in $\mathbb{F}_{p}[X]$, then $N(\alpha)=\alpha^{p+1} \equiv 1(\bmod p)$.

Therefore :

$$
\begin{aligned}
s_{p+1}(a) & \equiv \alpha^{p+1}+\alpha^{-(p+1)} \\
& \equiv N(\alpha)+N\left(\alpha^{-1}\right) \\
& =2 \\
& =s_{0}(a)(\bmod p) \text { and } \pi \text { divides } p+1 .
\end{aligned}
$$

If $f(X)$ is a primitive polynomial modulo $p$, then $\alpha$ generates the multiplicative group $\left(A^{*},.\right)$ with cardinal order $p^{2}-1$ and $\pi$ is exactly $\pi=p+1$.

Corollary 2.3 For every integer e such that $\operatorname{gcd}(e, \pi)=1$, where the gcd is the greatest common divisor, the map :

$$
\begin{aligned}
\text { Luc }_{e}: \mathbb{F}_{p} & \longrightarrow \mathbb{F}_{p} \\
a & \longmapsto s_{e}(a)
\end{aligned}
$$

is a one-one correspondence.

Proof: Indeed, since $\operatorname{gcd}(e, \pi)=1$, then we can use the Euclid algorithm to calculate the inverse $d$ of $e$ modulo $\pi$. That means there exists an integer $k$ such that $d e=1+k \pi$. Hence :

$$
\begin{aligned}
s_{d}\left(s_{e}(a)\right) & \equiv s_{d e}(a) \\
& \equiv s_{1+k \pi}(a) \\
& \equiv s_{1}(a) \\
& \equiv a(\bmod p) .
\end{aligned}
$$

Lemma 2.4 Let $e \in \mathbb{N}$ such that $\operatorname{gcd}(e, \pi)=1$ and let $c=s_{e}(a)$. Then $p$ does not divide $c^{2}-4$ if and only if $p$ does not divide $a^{2}-4$ and $\left(\frac{a^{2}-4}{p}\right)=\left(\frac{c^{2}-4}{p}\right)$.

Proof: Since $a \equiv s_{d}(c)(\bmod p)$, where $d$ is the inverse of $e$ modulo $\pi$, it suffices to show that if $\left(\frac{a^{2}-4}{p}\right)=1$, then $\left(\frac{c^{2}-4}{p}\right)=1$ too.

So, assume that $\left(\frac{a^{2}-4}{p}\right)=1$, then $\alpha \in \mathbb{F}_{p}$. Thus, $\alpha^{e} \in \mathbb{F}_{p}$ and $f_{c}(X)=X^{2}-c X+1$ splits in $\mathbb{F}_{p}[X] ; f_{c}(X) \equiv\left(X-\alpha^{e}\right)\left(X-\alpha^{-e}\right)(\bmod p)$. Therefore, i.e., $\left(\frac{c^{2}-4}{p}\right)=1$.

\subsection{Computation Method and analysis}

Lemma 2.5 For every integer $n$, set $s_{n}:=s_{n}(a)(\bmod p)$. Then

$$
\left\{\begin{array}{llll}
i) & s_{2 n} & \equiv & s_{n}^{2}-2 \\
\text { ii) } & s_{2 n+1} & \equiv & s_{n} s_{n+1}-a
\end{array}\right.
$$


Proof: Let $n$ and $m$ be two integers.

$$
\begin{aligned}
s_{n} s_{m} & \equiv\left(\alpha^{n}+\alpha^{-n}\right)\left(\alpha^{m}+\alpha^{-m}\right)(\bmod p) \\
& \equiv\left(\alpha^{n+m}+\alpha^{-n-m}\right)+\left(\alpha^{n-m}+\alpha_{2}^{-n+m}\right) \\
& \equiv s_{n+m}+s_{n-m}(\bmod p) .
\end{aligned}
$$

Therefore,

$s_{n+m} \equiv s_{n} s_{m}-s_{n-m}(\bmod p)$.

In particular, we have i) and ii).

Let $k=2^{r} m$, where $m$ is an odd integer. To compute $s_{k}$, first we compute $s_{m}$, then $s_{2 m}$ :

$$
\begin{aligned}
& s_{2 m} \equiv s_{m}^{2}-2 \\
& s_{4 m} \equiv s_{2 m}^{2}-2 \\
& \quad \cdots \cdots \\
& s_{k} \equiv s_{2^{r-1} m}^{2}-2 .
\end{aligned}
$$

Then to compute $s_{k}$, we need $r$ multiplications modulo $p$ and we need $s_{m}$.

Let $m=\sum_{i=0}^{l-1} k_{i} 2^{l-1-i}$. For every $0 \leq i<l-1$, let $f_{i+1}=2 f_{i}+k_{i+1}$ and $f_{0}=k_{0}$. Then $f_{l-1}=k$. For $0 \leq i<l-1$ and assume that, $s_{f_{i-1}}$ and $s_{f_{i-1}+1}$ are computed. Then

$$
\begin{aligned}
& \text { if } k_{i}=0 \text {, then }\left\{\begin{array}{ccc}
s_{f_{i}} & \equiv & s_{2 f_{i-1}} s_{f_{i-1}}^{2}-2 \\
s_{f_{i}+1} & \equiv & s_{2 f_{i-1}+1} \equiv s_{f_{i-1}}(a) s_{f_{i-1}+1}-a
\end{array}\right. \\
& \text { if } k_{i}=1 \text {, then }\left\{\begin{array}{ccc}
s_{f_{i}} & \equiv & s_{2 f_{i-1}+1} \equiv s_{f_{i-1}} s_{f_{i-1}+1}-a \\
s_{f_{i}+1} & \equiv & s_{2\left(f_{i-1}+1\right)} \equiv s_{f_{i-1}+1}^{2}-2
\end{array}\right.
\end{aligned}
$$

Computation Algorithm. This method warrant that $s_{k}$ can be computed in about the same length of time as the $k^{t h}$ power is computed in the RSA method. But in the computation of $s_{m}$, having to compute two numbers at each stage does slow the computation down a little, but there are optimizations in the calculation which mean that the total amount of computation is only about half less than the amount needed for the RSA system. Therefore, to compute $s_{k}(a)$, the total number of multiplications modulo $p$ is $\log _{2}(k)$.

\section{Main results}

In this section we describe some applications of Lucas sequences, in more details : Lucas multi-receiver encryption scheme and Lucas Diffie-Hellman key exchange extended to a group of communications. Through this section, $n=p q$ is an RSA integer, $a \in \mathbb{Z}$ sush that $\operatorname{gcd}(n, a)=1$, $\left(s_{n}\right)_{n}$ is the Lucas sequence generated by $f=x^{2}-a x+1$, and $f$ is a primitive polynomial over $\mathbb{F}_{p}$ and $\mathbb{F}_{q}$.

\subsection{Multi-receiver encryption scheme}

In this section, we propose a multi-receiver encryption scheme based on Lucas sequences and Chines Remainder Theorem. Our scheme consists of two procedures: Encryption procedure and decryption procedure. 


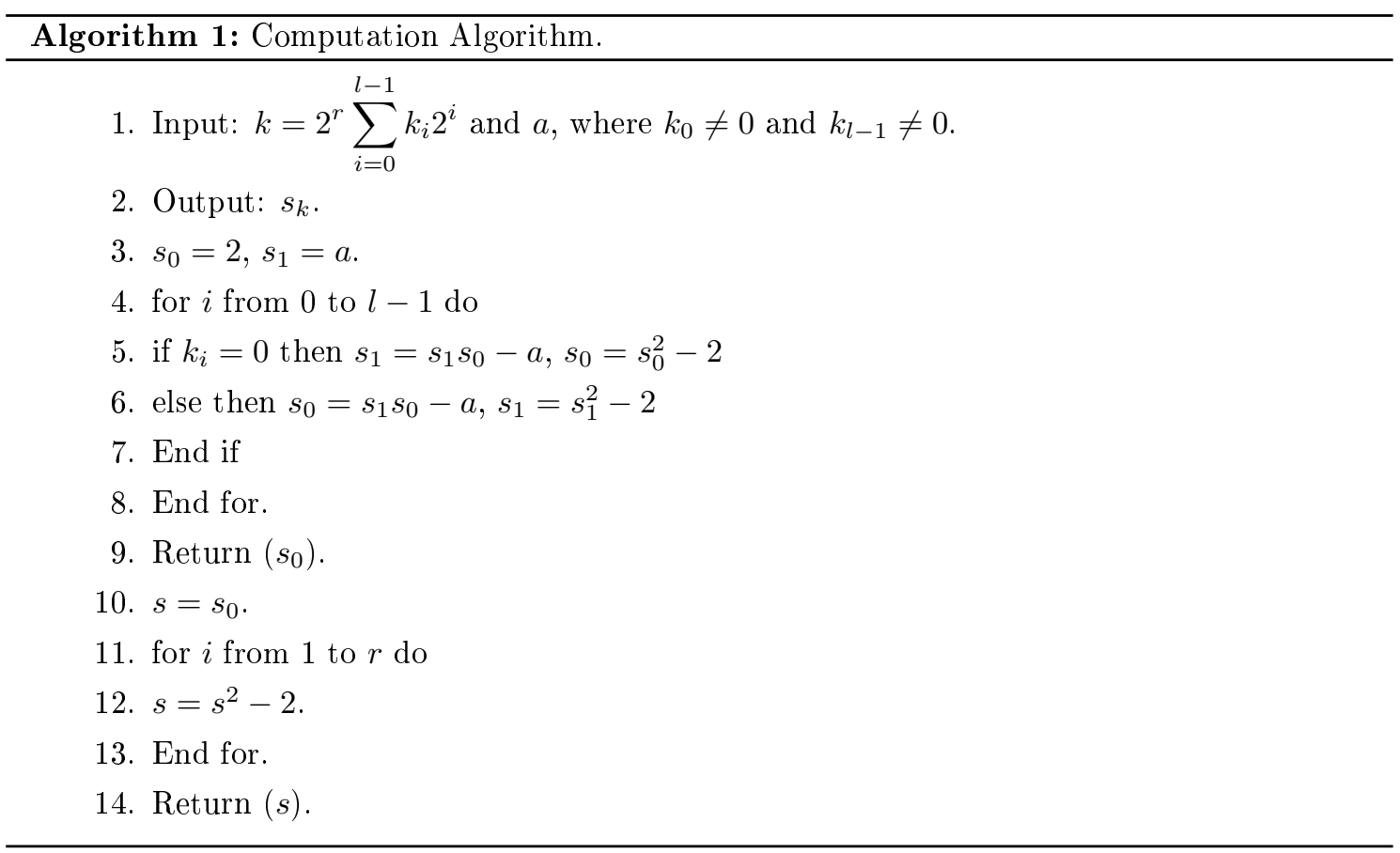

1. Keys generation : First, assume that there are $r+1$ users. Any user selects $s \leq r$ authorized receivers $u_{1}, \cdots, u_{s}$ such that each one has one public key pair $\left(n_{i}, e_{i}\right)$, where every $n_{i}=p_{i} q_{i}$ is an RSA integer, $e_{i}$ is coprime to $\left(p_{i} \mp 1\right)\left(q_{i} \mp 1\right)$ and $n_{1}, \cdots, n_{r}, n_{r+1}$ are pairwise coprime.

2. Encryption procedure : A sender uses all $s$ receivers public keys to encrypt the message and then broadcasts the ciphertext to the $s$ authorized receivers as follows :

(a) Assume that he needs to send a message $0<m<N$ to the $s$ legitimate receivers, where $N$ is the minimum of all $n_{i}$.

(b) Then for every $i=1, \ldots, s$, he calculates $c_{i}=s_{e_{i}}(m)$. As $n_{1}, \cdots, n_{s}$ are public and pairwise coprime.

(c) He uses the Chines Remainder Theorem to calculate the integer $c$ such that for every $i=1, \ldots, s, c \equiv c_{i}\left(\bmod n_{i}\right)$.

(d) Then He broadcasts the ciphertext $c$ to the $s$ receivers.

3. Decryption procedure : When a autorized receiver $i$ receives the ciphertext $c$, he will compute his private key $d_{i}$ as follows :

(a) First he calculates $c_{i} \equiv c\left(\bmod n_{i}\right), \epsilon_{p_{i}}=\left(\frac{c_{i}^{2}-4}{p_{i}}\right)$ and $\epsilon_{q_{i}}=\left(\frac{c_{i}^{2}-4}{q_{i}}\right)$.

(b) He calculates his private key $d_{i}$ :

the inverse of $e_{i}$ modulo $\pi=\left(p_{i}-\epsilon_{p_{i}}\right)\left(q_{i}-\epsilon_{q_{i}}\right)$.

(c) Finally, he calculates $m \equiv s_{d_{i}}\left(c_{i}\right)\left(\bmod n_{i}\right)$.

Proof :

Encryption :

Indeed, in order to calculate the ciphertext $c$, for every $i=1, \cdots, s$, the sender will calculate 
$M_{i}=\prod_{j=1, j \neq i}^{s} n_{j}, y_{i}$ its inverse modulo $n_{i}$ and $c=\sum_{i=1}^{s} c_{i} y_{i} M_{i}$.

As $y_{i} M_{i} \equiv 1\left(\bmod n_{i}\right)$ and for every $j \neq i, y_{i} M_{i} \equiv 0\left(\bmod n_{j}\right)\left(\right.$ since $n_{j}$ divides $\left.M_{i}\right)$, for every $i=1, \ldots, s, c \equiv c_{i}\left(\bmod n_{i}\right)$.

Decryption :

For the decryption phase, as $c_{i} \equiv s_{e_{i}}(m)\left(\bmod n_{i}\right)$ and $g c d(e, \pi)=1,\left(\frac{c_{i}^{2}-4}{p}\right)=\left(\frac{m^{2}-4}{p}\right)$ and $\left(\frac{c_{i}^{2}-4}{q}\right)=\left(\frac{m^{2}-4}{q}\right)$.

Thus,

$$
\begin{aligned}
s_{d_{i}}\left(c_{i}\right) & \equiv s_{d_{i}}\left(s_{e_{i}}(m)\right. \\
& \equiv s_{d_{i} e_{i}}(m) \\
& \equiv m\left(\bmod n_{i}\right) .
\end{aligned}
$$

\section{Analysis :}

Now, we will discuss security, computation cost and the anonymity of receivers of the proposed scheme. The anonymity means that the identities of receivers can be protected. We will compare the computation cost of our scheme and other existing schemes.

\subsubsection{Security}

Since this scheme uses "LUC" cryptosystem, the security of the scheme is the same as monocast "LUC" cryptosystem. Namely, if Oscar wants to decrypt the encrypted message $c$, first of all he needs to break the LUC cryptosystem, which is cryptographically equivalent to the security of $\operatorname{RSA}[17,2]$.

\subsubsection{Anonymity of Receivers}

When a sender broadcasts the ciphertext nobody in the group except the sender knows who is the receiving end.

\subsection{Diffie-Hellmane Key exchange extended to a group of communica- tion}

The standard Diffie-Hellman key exchange was first proposed in 1976 [20]. After that there have been efforts to extend its simplicity to a group setting. The main motivating factor is the increasing popularity of various types of group applications and the need of doing it securely. Since key distribution is the cornerstone of secure group communication, it has naturally received a lot of attention. Notable solutions have been proposed but some of theme are only of theoretical interest, while the security of some others remains unproven (See for example $[12,8,3,13]$ ). In this paper, a natural extension of Diffie-Hellman key exchange, based on Lucas sequences is proposed, see Figure 1.

Let $n=p q$ be an RSA integer, $a$ be an integer such that $X^{2}-a X+1$ is a primitive polynomial modulo $p$ and modulo $q$ too.

Assume that $r$ users $u_{1}, \cdots, u_{r}$, whose have access to a public key data $(n, a)$ and each one has his private key $x_{i}$, want to agree on a shared secret key. Then :

- The first user $u_{1}$ makes public $y_{1}^{(1)}=s_{x_{1}}(a)$. 
- The second one $u_{2}$, makes public $\left(y_{1}^{(2)}, y_{2}^{(2)}, y_{3}^{(2)}\right)$, where :

$$
\begin{aligned}
& y_{1}^{(2)}=s_{x_{2}}(a), \\
& y_{2}^{(2)}=s_{x_{1}}(a), \\
& y_{3}^{(2)}=s_{x_{2}}\left(y_{1}\right) .
\end{aligned}
$$

The notation $y_{i}^{j}$ means that this quantity is published by the $j^{\text {th }}$ user.

— The third makes public $\left(y_{1}^{(3)}, y_{2}^{(3)}, y_{3}^{(3)}, y_{4}^{(3)}\right)$, where :

$$
\begin{aligned}
& y_{1}^{(3)}=s_{x_{3}}\left(y_{1}^{2}\right), \\
& y_{2}^{(3)}=s_{x_{3}}\left(y_{2}^{2}\right), \\
& y_{3}^{(3)}=y_{3}^{2}, \\
& y_{4}^{(3)}=s_{x_{3}}\left(y_{3}^{2}\right) .
\end{aligned}
$$

- Once the $i^{t h}$ user makes public the tuple $\left(y_{1}^{(i)}, \cdots, y_{i+1}^{(i)}\right)$.

The $(i+1)^{t h}$ makes public $\left(y_{1}^{(i+1)}, \cdots, y_{i+2}^{(i+1)}\right)$, where for every $j=1, \cdots i$, :

$$
\begin{aligned}
& y_{j}^{(i+1)}=s_{x_{i+1}}\left(y_{j}^{(i)}\right), \\
& y_{i+1}^{(i+1)}=y_{i+1}^{(i)}, \\
& y_{i+2}^{(i+1)}=s_{x_{i+1}}\left(y_{i+1}^{(i)}\right) .
\end{aligned}
$$

- Finally, the last user $u_{n}$ makes public $\left(y_{1}^{(n)}, \cdots, y_{n}^{(n)}\right)$ and keeps secret the common private key $y=y_{n+1}^{(n)}=s_{x_{i}}\left(y_{i}^{(n)}\right)$.

- Each user $u_{i}, i=1, \cdots n-1$ can calculates the same value $y=s_{x_{i}}\left(y_{i}^{(n)}\right)$. The common private key is $y=y_{n+1}^{(n)}$. Indeed,

\section{Computation analysis:}

$$
\begin{aligned}
y & =s_{x_{n}}\left(y_{n}^{(n-1)}\right) \\
& =s_{x_{n}}\left(s_{x_{n-1}}\left(\ldots\left(s_{x_{1}}(a)\right) \ldots\right)\right) \\
& =s_{x_{n} x_{n-1} \ldots x_{1}}(a) .
\end{aligned}
$$

We compare the performance monocast "LUC" encryption and L. Harn et al. scheme [11] with the presented scheme. From Table 1 one can see that proposed scheme is more computationally efficient than the scheme proposed in [11], see Table 1.

\section{Conclusion}

In this paper, we introduced a new multi-receiver encryption scheme which provides the anonymity of receivers. Our scheme is more efficient than prevailing schemes in terms of computational cost. The security of this scheme is of equal strength as monocast LUC encryption. The comparison with others results and the security analyses clearly show that the proposed method provides anonymity of receivers and a good computational cost. Each receiver only needs to keep one private key and uses this private key to decrypt the ciphertext. 


\section{Acknowledgments}

The authors extend their appreciation to the Deanship of Scientific Research at King Khalid University for funding this work through General Research Project under grant number (G.R.P86-38).

\section{References}

[1] A. Fiat and M. Naor, "Broadcast encryption," in Proceeding of Advances Cryptology - Crypto, LNCS 839, pp. 480-491, Springer-Verlag, California, USA, Aug. 1994.

[2] Chi-Sung Laih, Fu-Kuan Tu, Wen-Chun Tai, On the security of the Lucas function, Information Processing Letters 53(1995), pp 243-247.

[3] Chin Chen Chang, Tzong Chen Wu, and C.P. Chen. The Design Of A Conference Key Distribution System. In Advances in Cryptology -AUSCRYPT'92, Lecture Notes in Computer Science, pages 467-474. Springer- Verlag, Berlin Germany, December 1992.

[4] C. I. Fan, L. Huang, and P. Ho, "Anonymous multi-receiver identity-based encryption," IEEE Transations on Computers, vol. 59, no. 9, pp. 1239-1249, Sep. 2010.

[5] D. Bleichenbacherl, W. Bosma, and K. Lenstra, Some Remarks on Lucas-Based Cryptosystems, Advances in Cryptology - CRYPT0 '95, LNCS 963, pp. 386-396, 1995

[6] Douglas R. Stinson, Cryptography Theory and Practice, Third edition 2006, Chapman, Hall/CRC, Taylor and Francis Group.

[7] G. H. Chiou and W. T. Chen, "Secure broadcasting using the secure lock" IEEE Transations on Software Engineering, vol. 15, no. 8, pp. 929-934, Aug. 1989.

[8] Hugh Harney, Carl Muckenhirn, and Thomas Rivers. Group Key Management Protocol (GKMP) Architecture. INTERNET-DRAFT, September 1994.

[9] H. Ghodosi, J. Pieprzyk, and R. Safavi-Naini, 'Dynamic threshold cryptosystems: a new scheme in group oriented cryptography," in Proceeding of Pragocrypt'96: the 1st International conference on the Theory and Applications of Cryptology, pp. 370-379, Prague, Czech Republic, Sep. 1996.

[10] L. El Fadil, A Public-Key Cryptosystem Based on Lucas Sequences, Palestine Journal of Mathematics Vol. 1(2) (2012) 148-152.

[11] L. HARN, C.- C. CHANG, and H.-L. WU, "An Anonymous Multi-Receiver Encryption Based on RSA", Journal of Network Security, Vol.15, No.4, PP.307-312, July 2013.

[12] M. Steiner, G. Tsudik and M. Waidner, Diffie-Hellman Key Disrtribution Extended to Group Communication, Proceding of the 3rd ACM conference on computer and communications security, March 14-16 (1996), new Delhi.

[13] M. Burmester and Y. Desmedt. A Secure And Efficient Conference Key Distribution System. In I.B. Damgard, editor, Advances in Cryptology, EUROCRYPT'94, Lecture Notes in Computer Science. Springer-Verlag, Berlin Germany, 1994.

[14] M. Bellare, A. Boldyreva, and S. Micali, 'Public-key encryption in a multi-user setting: security proofs and improvements," in Proceeding of Advances Cryptology - Eurocrypt 2000, LNCS 1807, pp.259-274, Springer-Verlag, Bruges, Belgium, May 2000.

[15] P. Ribenboim, The Little Book of Big Primes, Springer-Verlag, 1991.

[16] P. Smith. LUC Public Key System : A secure Alternative to RSA. Dr. Dobb's Journal, January 1993.

[17] P. Smith and M. J. J. Lennon, LUC : A new public key system. In Proc. of the Ninth IFIP Int. Symp. on Computer Security, p. 103-117, 1993. 
[18] S. Berkovits, "How to broadcast a secret," in Proceeding of Advances Cryptology - Eurocrypt'91, LNCS 547, pp. 535-541, Springer-Verlag, Brighton, UK, Apr.1991.

[19] S. Berkovits, "How to broadcast a secret," in Proceeding of Advances Cryptology - Eurocrypt'91, LNCS 547, pp. 535-541, Springer-Verlag, Brighton, UK, Apr.1991.

[20] W. Diffie and M. E. Hellman, New directions in cryptography, IEEE Trans. Inform. Theory, vol. IT-22, (1976), pp. 644-654.

[21] X. Du, Y. Wang, J. Ge, and Y. M. Wang, "An ID-based broadcast encryption scheme for key distribution," IEEE Transations Broadcast, vol. 51, no. 2, pp. 264-266, June 2005.

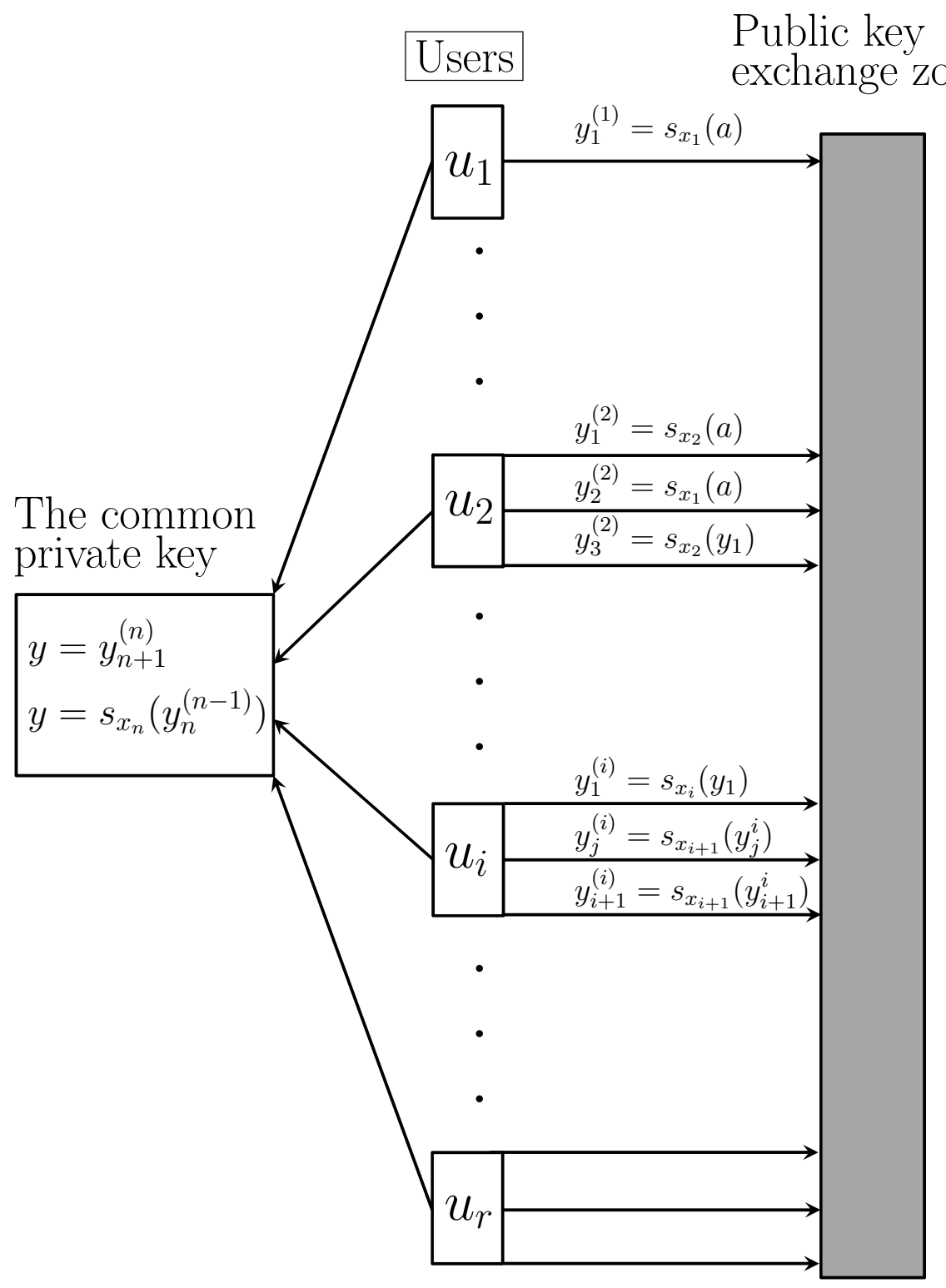

Figure 1: Diffie-Hellmane Key exchange extended to a group of communication. 


\begin{tabular}{|l|c|c|c|}
\hline & Monocast LUC encryption & L. Harn, et al. scheme & Our scheme \\
\hline Sender & $n / 2 \times T_{E} 1024$ & $n \times T_{E} 1024$ & $n / 2 \times T_{E} \_1024$ \\
\hline Receiver & $T_{D}$ & $T_{D}$ & $T_{D}$ \\
\hline Rounds of transmission & $n$ & 1 & 1 \\
\hline Anonymity of receivers & No & Yes & Yes \\
\hline
\end{tabular}

Table 1: Comparison results among different schemes. 